\title{
CX3CR1-microglia mediates neuroinflammation and blood pressure regulation in the nucleus tractus solitarii of fructose-induced hypertensive rats
}

Chiu-Yi Ho ${ }^{1,2+}$, Yu-Te Lin ${ }^{2,3,4,5 \dagger}$, Hsin-Hung Chen ${ }^{1}$, Wen-Yu Ho ${ }^{6,7}$, Gwo-Ching Sun ${ }^{8,9}$, Michael Hsiao ${ }^{10}$, Pei-Jung Lu ${ }^{11}$, Pei-Wen Cheng ${ }^{1,2^{*}}$ and Ching-Jiunn Tseng ${ }^{1,2,12^{*}}$ (D)

\begin{abstract}
Background: Inflammation is a common pathophysiological trait found in both hypertension and cardiac vascular disease. Recent evidence indicates that fractalkine (FKN) and its receptor CX3CR1 have been linked to inflammatory response in the brain of hypertensive animal models. Here, we investigated the role of CX3CR1-microglia in nitric oxide (NO) generation during chronic inflammation and systemic blood pressure recovery in the nucleus tractus solitarii (NTS).

Methods: The hypertensive rat model was used to study the role of CX3CR1-microglia in NTS inflammation following hypertension induction by oral administration of 10\% fructose water. The systolic blood pressure was measured by tail-cuff method of non-invasive blood pressure. The CX3CR1 inhibitor AZD8797 was administered intracerebroventricularly (ICV) in the fructose-induced hypertensive rat. Using immunoblotting, we studied the nitric oxide synthase signaling pathway, NO concentration, and the levels of FKN and CX3CR1, and pro-inflammatory cytokines were analyzed by immunohistochemistry staining.

Results: The level of pro-inflammatory cytokines IL-1 $\beta$, IL-6, TNF- $a$, FKN, and CX3CR1 were elevated two weeks after fructose feeding. AZD8797 inhibited CX3CR1-microglia, which improved the regulation of systemic blood pressure and NO generation in the NTS. We also found that IL-1 $\beta, \mathrm{IL}-6$, and TNF-a levels were recovered by AZD8797 addition.

Conclusion: We conclude that CX3CR1-microglia represses the nNOS signaling pathway and promotes chronic inflammation in fructose-induced hypertension. Collectively, our results reveal the role of chemokines such as IL-1 $\beta$, IL-6, and TNF-a in NTS neuroinflammation with the involvement of FKN and CX3CR1.
\end{abstract}

Keywords: Nucleus tractus solitarii, Fractalkine, CX3CR1, Hypertension, Inflammation

* Correspondence: pwcheng@vghks.gov.tw; cjtseng@vghks.gov.tw ${ }^{+}$Chiu-Yi Ho and Yu-Te Lin indicates co-first authors.

${ }^{1}$ Department of Medical Education and Research, Kaohsiung Veterans General Hospital, Kaohsiung 81300, Taiwan

Full list of author information is available at the end of the article

\section{Introduction}

Cardiovascular disease is a complicated condition that affects metabolism and blood pressure [1,2]. Epidemiological study showed that unhealthy dietary habit contributes to increased blood pressure, hyperglycemia, hyperlipidemia, and neuroinflammation [3]. Despite recent studies showing that excessive fructose

C C The Author(s). 2020 Open Access This article is licensed under a Creative Commons Attribution 4.0 International License, which permits use, sharing, adaptation, distribution and reproduction in any medium or format, as long as you give appropriate credit to the original author(s) and the source, provide a link to the Creative Commons licence, and indicate if changes were made. The images or other third party material in this article are included in the article's Creative Commons licence, unless indicated otherwise in a credit line to the material. If material is not included in the article's Creative Commons licence and your intended use is not permitted by statutory regulation or exceeds the permitted use, you will need to obtain permission directly from the copyright holder. To view a copy of this licence, visit http://creativecommons.org/licenses/by/4.0/ The Creative Commons Public Domain Dedication waiver (http://creativecommons.org/publicdomain/zero/1.0/) applies to the data made available in this article, unless otherwise stated in a credit line to the data. 
consumption leads to pro-inflammatory response and microglia activation, the role of pro-inflammatory factor in the nucleus tractus solitarii (NTS) is not wellunderstood $[4,5]$. In addition, we previously showed that the brainstem NTS, part of the brain which integrates signals from the peripheral carotid sinus and aortic arch to regulate systemic blood pressure, decreases production of nitric oxide $(\mathrm{NO})$ during fructose-induced hypertension [6]. However, the underlying mechanism involving NO during chronic inflammation in the NTS is not yet clarified. Therefore, it is essential to investigate the role of FKN and its receptor CX3CR1 after hypertension induced by fructose.

C-X3-C motif chemokine receptor 1 (CX3CR1) is a chemokine receptor that binds to its ligand $\mathrm{C}-\mathrm{X} 3-\mathrm{C}$ motif chemokine ligand 1 (CX3CL1), also known as fractalkine (FKN), and is found in the leukocytes, brain, spinal cord, and retina $[7,8]$. Both FKN and CX3CR1 have been detected in vivo in the brainstem NTS. CX3CR1 is characterized as microglia biomarker. According to Bhaskar et al., CX3CR1 is the potential target for studying metabolic syndrome $[9,10]$. The FKNCX3CR1 interaction is associated with crosstalk between neurons and microglia. Previous study has suggested that CX3CR1 may be involved in neurodegenerative diseases such as multiple sclerosis [11], Alzheimer's disease [5], spinal cord injury, and traumatic brain injury [12]. Ruchaya et al. reported that FKN microinjection produces cardiovascular response in the NTS of normal rats [13]. However, the function of CX3CR1 during neurogenic hypertension progression in the NTS is ambiguous. On the other hand, NO is a gas molecule that is involved in various functions in the dorsal brainstem NTS such as vasodilation, baroreflex, sympathetic nerve activity, and most importantly blood pressure maintenance [14]. It is essential that the autonomic response to combine afferent signals be integrated from the peripheral nervous system for optimal adjustment of sympathetic activity. In the clinical aspect, overactivity of the sympathetic nervous system contribute to the development of hypertension [15]. This study aims to examine the NTS cardiovascular effect and whether increased pro-inflammatory cytokines such as CX3CR1 has impact on $\mathrm{NO}$ production.

There is increasing evidence that inflammation in the brain is associated with excessive fructose intake. Such inflammatory response poses a threat to the brain and microglia cells which reside in the brain that act upon the threat to clear debris and repair damaged neural tissue. However, chronic inflammation in the brain activates microglia cells to release pro-inflammatory cytokines that are harmful to brain tissue $[16,17]$. Furthermore, fructose, in particular, induces hypertension in vivo which strongly correlates with chronic brain inflammation [18-20]. Xu et. al. reported that fructose increases blood pressure, and elevates transcription of pro-inflammatory cytokines, IL-1 $\beta$, IL-6, and TNF- $\alpha$ in the brain after 8 weeks of fructose consumption [5].

Our previous results suggest that fructose reduces central NO production, increases systemic blood pressure and renal sympathetic nerve activity [14, 21, 22]. The signaling pathway, PI3K-Akt-ERK1/2-nNOS (neuronal nitric oxide synthase) pathway, is shown to regulate nNOS phosphorylation in the NTS [23]. Additionally, we found that fructose disrupted the Akt-ERK1/2nNOS signaling pathway $[6,21]$. Short-term or 2 weeks of fructose feeding increases TNF- $\alpha$ in the brain [4]. We previously reported that prolonged fructose feeding time period induces blood pressure [22]. These results support the idea that blood pressure increase triggered by dietary fructose is closely linked to pro-inflammatory cytokines in the brain. We therefore speculate that CX3CR1-microglia may affect NO production to modulate NTS inflammation, and selective CX3CR1 inhibitor AZD8797 may be able to treat the NTS proinflammatory response. We observed that proinflammatory cytokines and NO production were suppressed through abolishing the Akt-ERK1/2-nNOS signaling pathway. Finally, microglia activation in the NTS requires CX3CR1 to promote fructose-induced hypertension progression.

\section{Methods}

\section{Animal care}

All procedures were reviewed and approved by the Institute of Animal Care and Use Committee at the Kaohsiung Veterans General Hospital (VGHKS, Kaohsiung, Taiwan). Wistar-Kyoto rats (WKY) were purchased from National Laboratory Animal Center (NLAC, Taipei, Taiwan), housed in the VGHKS animal center with light-controlled cycle of 12-h light and 12-h darkness.

\section{Experimental design}

WKY animals were divided into five groups. Each consisted of six to eight animals in total: (1) control rats received tap water (Ctrl); (2) hypertensive rats received $10 \%$ fructose water for 2 or 4 weeks $(\mathrm{F} 2 \mathrm{w}, \mathrm{F} 4 \mathrm{w})$; (3) vehicle-control rats were fed with tap water for 2 weeks, followed by osmotic pump implantation (14 days, $0.5 \mu \mathrm{L} /$ h) filled with $30 \%$ 2-hydroxypropyl- $\beta$-cyclodextrin (HP$\beta$-CD) (Santa Cruz, Dallas, TX, USA) and continued tap water feeding simultaneously in the subsequent 2 weeks (V-Ctrl); (4) vehicle-fructose rats received 10\% fructose water for 2 weeks, followed by osmotic pump implantation (14 days, $0.5 \mu \mathrm{L} / \mathrm{h}$ ) filled with $30 \%$ 2hydroxypropyl- $\beta$-cyclodextrin (HP- $\beta-\mathrm{CD})$ and continued $10 \%$ fructose water simultaneously in the subsequent 2 weeks (V-Fru); (5) finally, the fructose-AZD8797 rats 
received $10 \%$ fructose water for 2 weeks, followed by osmotic pump implantation (14 days, $0.5 \mu \mathrm{L} / \mathrm{h}$ ) filled with AZD8797 (Axon Medchem, Groningen, Netherlands) solution and continued 10\% fructose water simultaneously in the subsequent 2 weeks (Fru-AZD).

\section{Non-invasive blood pressure (NIBP) measurement}

The systolic blood pressure (SBP) was measured by using the tail-cuff method (CODA, Kent Scientific, Torrington, CT, USA). Operating procedures were carried out according to the manufacturer's manual. Briefly, the WKY rats were trained in a rodent holder and placed on the warm plate $\left(35^{\circ} \mathrm{C}\right)$ for three times before the experiment began. Measurements were taken 15 times, restricted to $40 \mathrm{~min}$ and below for the entire procedure, and systolic blood pressure data were collected at the end of the experiment. To prevent blood pressure fluctuation as a result of circadian rhythms, the data were collected between 09:00-12:00 am, coordinated universal time with + 08:00 offset for Taiwan time zone.

\section{Cerebrospinal fluid (CSF) and serum sample collection and measurement}

After a 16-hr fast, the rats were anesthetized with isoflurane $\left(2 \%\right.$ mixed with $\left.\mathrm{O}_{2}\right)$, and blood samples were collected using serum separation tubes. To collect serum, the tubes were centrifuged $(3000 \times g)$ at $4{ }^{\circ} \mathrm{C}$ for $10 \mathrm{~min}$. The CSF was collected in a $1.5-\mathrm{mL}$ Eppendorf tube from the fourth ventricle through an insulin needle. The tubes were centrifuged $(300 \times g)$ at $4{ }^{\circ} \mathrm{C}$ for $10 \mathrm{~min}$ to remove residual blood. Immediately, all samples were dispensed and frozen at $-80^{\circ} \mathrm{C}$ for biochemical assays. All serum parameters were measured by VITROS 350 Chemistry System (Ortho Clinical Diagnostics, New Brunswick, NJ, USA).

\section{Intracerebroventricular administration procedure}

The three animal groups: V-Ctrl, V-Fru, and Fru-AZD underwent intracerebroventricular surgery. The osmotic pump (Alzet, Cupertino, CA, USA) which was filled with AZD8797solution, vehicle solution $30 \% \quad 2$ hydroxypropyl- $\beta$-cyclodextrin (HP- $\beta-C D)$, was immersed in normal saline for $16 \mathrm{~h}$ at $4{ }^{\circ} \mathrm{C}$ prior to surgery. During anesthetization, Atropine $0.05 \mathrm{mg} / \mathrm{kg}$, Zolile $40 \mathrm{mg} / \mathrm{kg}$, and Xylazine $10 \mathrm{mg} / \mathrm{kg}$ were placed on the stereotaxic instrument; the head was fixed with the cranium exposed. The position of the injection was located $1.5 \mathrm{~mm}$ lateral and caudal $0.8 \mathrm{~mm}$ from the bregma. After surgery, the rat tail systolic blood pressure was measured every week.

\section{Immunoblot analysis}

The brain was excised and the NTS regions (approximately $20 \mathrm{mg}$ ) were separated according to the rat brain in stereotaxic coordinates based on Paxinos and Watson, sixth edition in 2007 [24]. Briefly, the NTS was dissected by micropunch ( $1 \mathrm{~mm}$ inner diameter) from 1-mm-thick brainstem slice at the level of the obex under the microscope. To collect total protein, NTS tissue was homogenized using T-PER (Thermo Fisher, Waltham, MA USA) containing protease and phosphatase inhibitors cocktail at $4{ }^{\circ} \mathrm{C}$. Protein was quantitatively analyzed with Coomassie R-250 (Thermo Fisher, Waltham, MA USA), subjected to 4-20\% SDS gradient gel electrophoresis, transferred to PVDF membrane. The membranes were blocked with 5\% BSA in TBS/Tween-20 buffer (10 mM Tris, $150 \mathrm{mM} \mathrm{NaCl}, 0.1 \%$ Tween 20, pH 7.4), incubated with anti-p-Akt ${ }^{5473}$ (Cell signaling, 9271), anti-Akt (Cell signaling, 9271), anti-p-eNOS ${ }^{\text {S1177 }}$ (BD, 612393), antieNOS (BD, 610297), anti-p-nNOS ${ }^{\mathrm{S} 1416}$ (Abcam, ab5583), anti-nNOS (Millipore, 07-571), anti-p-ERK1/2 ${ }^{\mathrm{T} 202 / \mathrm{Y} 204}$ (Cell signaling, 9101), anti-ERK1/2 (Cell signaling, 9102), or $\beta$ actin (Millipore, MAB1501) antibody at 1:1000 dilution in phosphate-buffered saline (PBS) Tween-20 with $5 \%$ BSA at $4{ }^{\circ} \mathrm{C}$ overnight. Peroxidase-conjugated anti-mouse or antirabbit antibody (1:5000) was used as the secondary antibody. The proteins were visualized using enhanced chemiluminescence (ECL), Pico plus detection kit (Thermo Fisher, Waltham, MA, USA), and film. The films were captured by ChemiDoc $^{\text {mit }}$ MP Imaging System (Bio-Rad, Hercules, CA, USA) and analyzed with Image Lab $^{\text {tix }}$ Software (Bio-Rad, Hercules, CA, USA).

\section{Analysis of NO concentration in the NTS}

To determine NO concentration in the groups V-Ctrl, V-Fru, and Fru-AZD, the NTS protein lysate was deproteinized using a Microcon YM-30 filter unit (Millipore, Darmstadt, $\mathrm{GmbH}$ ) based on a previous method [6]. The total amount of $\mathrm{NO}_{x}$ in the samples were determined using modified chemiluminescence-based procedure and Sievers Nitric Oxide Analyzer purge system (NOA 280i, Sievers Instruments, Boulder, CO, USA) [25].The sample $(10 \mu \mathrm{L})$ was injected into reflux column containing 0.1 $\mathrm{mol} / \mathrm{L} \mathrm{VCl}_{3}$ in $1 \mathrm{~mol} / \mathrm{L} \mathrm{HCl}$ at $90^{\circ} \mathrm{C}$ to reduce nitrates and nitrites into NO. The $\mathrm{NO}_{x}$ was then combined with $\mathrm{O}_{3}$ produced by the analyzer to form $\mathrm{NO}_{2}$. The emission resulting from the excited $\mathrm{NO}_{2}$ was detected by a photomultiplier tube and digitally recorded $(\mathrm{mV})$. The values were then interpolated to standard curve of concurrently determined $\mathrm{NaNO}_{2}$ concentrations. The measurements were recorded in triplicate for each sample. The $\mathrm{NO}_{x}$ levels measured were corrected for the NTS protein concentration of the rats.

\section{Enzyme-linked immunosorbent assay (ELISA)}

The NTS of the brainstem were sectioned and homogenized with T-PER (Thermo Fisher, Waltham, MA, USA) containing protease and phosphatase inhibitors cocktail 


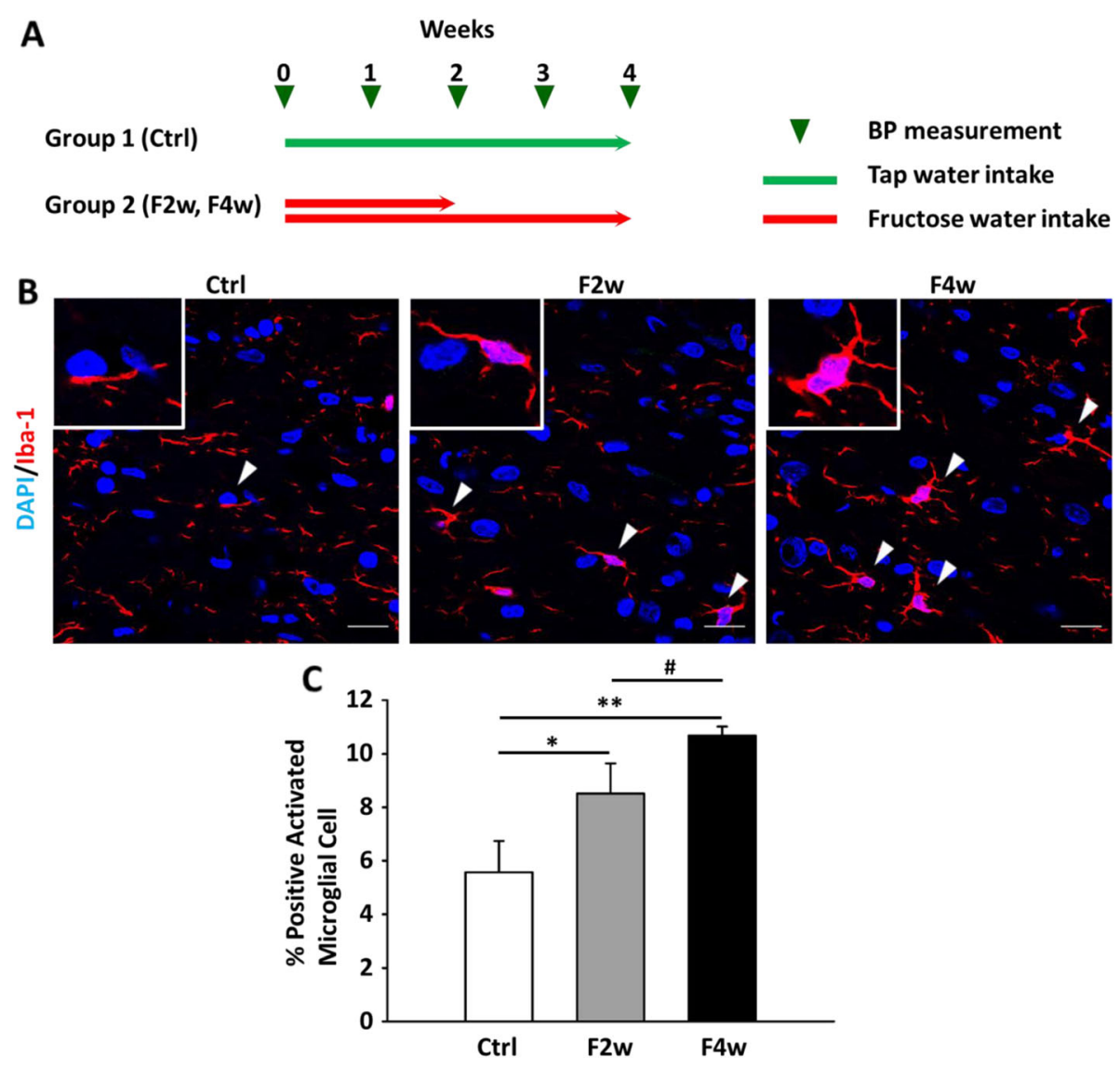

Fig. 1 Quantitative immunofluorescent analysis of microglial marker in the NTS following feeding with 10\% fructose. a Flowchart presented animal experimental design. $\mathbf{b}$ In situ qualitative analysis of the microglial marker Iba-1 by immunofluorescent staining. The arrowhead indicates activated microglial cells. The scale bar represents $20 \mu \mathrm{m}$. c Graphs depicting quantitative analysis of in situ positive cells in the NTS of WKY rats after fructose feeding. The percentage of the positive cells was determined by counting positive cells in each hemisphere of the NTS at $400 \times$ magnification. The values are presented as mean \pm SEM. One-way ANOVA with Bonferroni's post-hoc was performed for statistical analysis. ${ }^{*} P<$ 0.05 , ${ }^{* *} P<0.01$ compared to the control group, $\# P<0.05$ compared to fructose feeding for 4 weeks ( $n=6 \sim 8$ per group)

at $4{ }^{\circ} \mathrm{C}$. The total protein was harvested by grinding and centrifugation. The total protein content was quantitatively analyzed by Coomassie R-250 (Thermo Fisher, Waltham, MA, USA). The concentration of IL-1 $\beta$, IL-6, TNF- $\alpha$, and fractalkine of serum, CSF, or NTS protein lysate were measured by ELISA kit, performed according to the manufacturer's instruction (Cloud-Clone Corp, Katy, TX, USA). Expression values were detected by Anthos Zenyth 200rt Microplate Reader (Biochrom, Cambridge, UK). The final values were calculated and normalized to NTS protein mass.

\section{Immunofluorescent staining analysis}

The Ctrl, F2w, and F4w animals were perfused with saline, followed by $4 \%$ paraformaldehyde solution. The brainstem was harvested and immersed in 30\% sucrose solution until it was sunken to the bottom of the tube, and this procedure was repeated once. Brain stem sections $(5 \mu \mathrm{m})$ were blocked with $5 \%$ bovine serum albumin and $0.3 \%$ Triton
X-100 for $30 \mathrm{~min}$ at room temperature, incubated in primary antibody anti-Iba-1 (Wako, 019-19741) for $16 \mathrm{~h}$ at $4{ }^{\circ} \mathrm{C}$. After PBS wash, the sections were incubated in Alexa Flour 488 Goat anti-rabbit IgG (Thermo Fisher Scientific, Waltham, MA, USA) for $1 \mathrm{~h}$ under room temperature. The tissues were mounted in VECTASHIELD mounting medium containing DAPI (Vector Labs, Burlingham, CA, USA). The sections were analyzed under LSM 800 laser scanning mode of confocal microscope (Carl Zeiss MicroImaging, Jena, $\mathrm{GmbH})$. The images were acquired using $40 \mathrm{x}$ magnification (objective: Plan-APO $40 \mathrm{x} / 1.30$ Oil DIC (UV) VIS-IR ), image matrix of $1024 \times 1024$ pixel, pixel scale $0.156 \times 0.156 \mu \mathrm{m}$, and a depth of 8 bit. Z-stacked images were collected with $0.33-\mu \mathrm{m}$ slice distance for 15 slices in total.

\section{Immunohistochemistry staining analysis}

The brain stem sections $(5 \mu \mathrm{m})$ were blocked in $5 \%$ bovine serum albumin and $0.3 \%$ Triton $\mathrm{X}-100$ for $30 \mathrm{~min}$ 
at room temperature, incubated in primary antibody anti-IL-1 $\beta$ (Proteintech, 16806-1-AP) and anti-IL-6 (Proteintech, 21865-1-AP) in primary antibody diluent (ScyTek laboratories, Logan, UT, USA) for $16 \mathrm{~h}$ at $4{ }^{\circ} \mathrm{C}$. After PBS wash, the sections were incubated in Novolink Polymer solution (Leica Biosystems, Nussloch, $\mathrm{GmbH}$ ) for $10 \mathrm{~min}$ under room temperature. The tissues were stained in DAB chromogen at room temperature, analyzed by Olympus BX51 microscope (Olympus Tokyo, Japan) and Image Browser (Carl Zeiss, MicroImaging, Jena, $\mathrm{GmbH}$ ).

\section{Statistical analysis}

All data were expressed as mean \pm SEM at least three independent experiments. IBM SPSS Statistics 20 was used in this study. The blood pressure (BP) measurements (fructose-treated and no-treatment groups) were analyzed by one-way ANOVA for repeated measurements and Bonferroni's post-hoc tests. Oneway ANOVA with Scheffe's post-hoc comparison was applied to immunoblotting and immunohistochemistry stain. $P<0.05$ was considered statistically significant.

\section{Results}

Fructose consumption leads to higher blood pressure and central inflammation

Fructose feeding induces hypertension in rats, and data are analyzed at the second and fourth weeks. The systolic blood pressure, fasting blood glucose, high-density lipoprotein, and triglyceride content were increased in the fructose group compared to control. Proinflammatory cytokines IL- $1 \beta$ and IL- 6 and TNF- $\alpha$ significantly increased in serum and NTS after fructose administration, but FKN showed no increase in the serum after 4 weeks of fructose feeding. Interestingly, FKN in the CSF and NTS increased after 2 weeks of fructose feeding (Table 1). These data demonstrated that fructose consumption leads to higher blood pressure and central inflammation.

\section{Microglia mediate blood pressure regulation in the NTS in fructose-induced hypertension}

Previously, CX3CR1 has been shown to be a putative microglial marker which was implicated to regulate blood pressure and heart rate [13]. In this study, we determined microglia activation through Iba-1 staining in NTS after fructose administration. The activated microglia showed stronger IBA-1 signal and shorten processes in the NTS after 2 or 4 weeks of fructose feeding (Fig. 1a). We quantified the NTS-activated microglia for fructose and control groups (Fig. 1b). Based on these observations, we speculate that microglia may have begun to intervene in the NTS, affecting blood pressure in the early stage of fructose-induced hypertension.

The fractalkine is a sole ligand that couples to CX3CR1, existing in either membrane-anchored or soluble form. The membrane-anchored FKN (mFKN), soluble FKN (sFKN), and CX3CR1 were analyzed by immunoblotting (Fig. 2a). After 4 weeks of fructose feeding, the sFKN was increased in the NTS but not mFKN and CX3CR1. Next, the level of sFKN in the NTS was significantly higher compared to control after 4 weeks of fructose feeding (Fig. 2b). As a result, we speculate that soluble FKN may have begun to stimulate microglia activation in the NTS after fructose consumption, rather than mFKN.

Table 1 General characteristics of the fructose-induced WKY rats

\begin{tabular}{|c|c|c|c|}
\hline Parameter/group & Control & Fructose 2 weeks & Fructose 4 weeks \\
\hline Systolic blood pressure, mmHg & $109.8 \pm 1.8$ & $125.1 \pm 2.2^{* * *}$ & $144.5 \pm 1.1^{* * *}$ \\
\hline Fasting serum glucose, mg/dL & $85.3 \pm 1.8$ & $134.2 \pm 4.9^{* * *}$ & $147.0 \pm 4.0^{* * *}$ \\
\hline Fasting serum triglyceride, mg/dL & $82.7 \pm 1.2$ & $155.3 \pm 18.6^{* * *}$ & $177.8 \pm 26.6^{* * *}$ \\
\hline Fasting serum dHDL, mg/dL & $83.0 \pm 1.6$ & $75.5 \pm 4.5^{*}$ & $71.5 \pm 2.5^{*}$ \\
\hline Serum IL-1 $\beta, p g / m L$ & $27.93 \pm 5.13$ & $49.97 \pm 8.59^{*}$ & $74.90 \pm 7.07^{*}$ \\
\hline Serum IL-6, pg/mL & $3.44 \pm 0.46$ & $6.21 \pm 0.24^{*}$ & $10.02 \pm 1.69^{*}$ \\
\hline Serum TNF-a, pg/mL & $25.82 \pm 9.05$ & $74.06 \pm 5.62^{*}$ & $202.10 \pm 17.82^{*}$ \\
\hline Serum fractalkine, ng/mL & $0.38 \pm 0.06$ & $0.36 \pm 0.04$ & $0.43 \pm 0.05$ \\
\hline CSF fractalkine, ng/mL & $1.20 \pm 0.04$ & $1.49 \pm 0.07^{*}$ & $1.65 \pm 0.03^{*}$ \\
\hline NTS fractalkine, ng/mg & $4.06 \pm 0.17$ & $4.80 \pm 0.36^{*}$ & $5.98 \pm 0.38^{*}$ \\
\hline NTS IL-1 $\beta, \mathrm{pg} / \mathrm{mg}$ & $6.53 \pm 0.27$ & $9.17 \pm 0.32^{*}$ & $12.63 \pm 0.83^{*}$ \\
\hline NTS IL-6, pg/mg & $61.13 \pm 6.30$ & $115.48 \pm 3.30^{*}$ & $150.87 \pm 11.68^{*}$ \\
\hline NTSTNF-a, pg/mg & $1.15 \pm 0.24$ & $4.67 \pm 0.75^{*}$ & $12.17 \pm 0.41^{*}$ \\
\hline
\end{tabular}

Levels of systolic blood pressure; fasting serum glucose; fasting serum triglycerides; fasting serum dHDL; and quantitative ELISA of serum, CSF, or NTS areas for FKN, IL-1 $\beta$, IL- 6 , and TNF- $\alpha$ are presented as mean \pm SEM. One-way ANOVA with Bonferroni's post-hoc was performed for statistical analysis.

${ }^{*} P<0.05,{ }^{* * *} P<0.001$ compared to control group ( $n=6 \sim 8$ per group) 

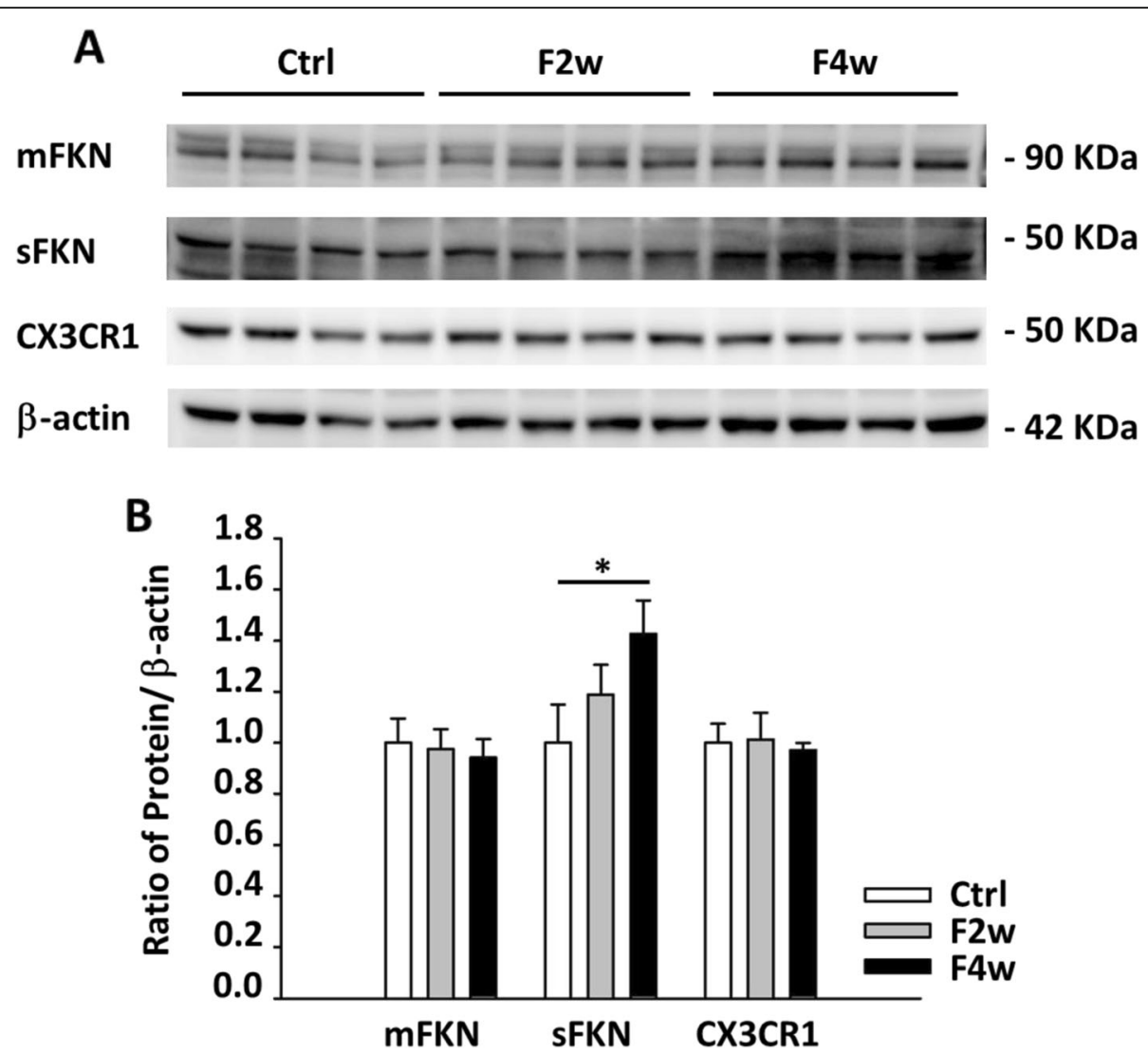

Fig. 2 Semi-quantitative protein expression of membrane-anchored FKN, soluble FKN, and CX3CR1 in the NTS after fructose feeding for 2 or 4 weeks. a The immunoblotting of FKN in the fructose and control animals after 4 weeks of fructose feeding. $\mathbf{b}$ Graphs depicting semi-quantitative analysis of protein expression level in NTS of WKY rats after fructose feeding. The values are presented as mean \pm SEM. One-way ANOVA with Bonferroni's post-hoc was performed for statistical analysis. ${ }^{*} P<0.05$ compared to the control group $(n=6 \sim 8$ per group)

\section{CX3CR1-microglia mediated systolic blood pressure improvement in the NTS}

To investigate whether CX3CR1-microglia was involved in systolic blood pressure regulation in fructose-induced hypertensive rats, CX3CR1 was inhibited by AZD8797 treatment. AZD8797 was injected into central intracerebroventricularly via osmotic minipump 2 weeks after hypertensive animals were established (Fig. 3a). Furthermore, the hypertensive animals treated with AZD8797 showed systolic blood pressure decreased to normotensive level 2 weeks after treatment began (Fig. 3b).

Our previous studies suggested that fructose-fed rats had reduced NO production in the NTS $[21,22]$. Therefore, we measured the NO level and found that NO production was improved in the NTS of AZD8797-treated animals. This suggests that CX3CR1-microglia in the NTS participates in NO and systemic blood pressure regulation (Fig. 3c). The increased NTS microglia activation may be involved in cardiovascular regulation that elicits a pressure effect and attenuates NO production through CX3CR1-microglia activation.

\section{AZD8797, CX3CR1 inhibitor, restored nNOS pathway in the NTS}

It was previously reported that the cardiovascular regulation effect is mediated by Akt signaling in normal rats [13]. However, our previous study showed that fructose intake causes defect of the PI3K-AktnNOS pathway $[6,21]$. To test the pathophysiological role of CX3CR1-microglia in the fructose-fed rat, we analyzed the Akt-nNOS pathway in the NTS using immunoblotting. The level of phosphorylated ERK1/2, Akt, and nNOS proteins may be recovered after AZD8797 treatment, and phosphorylated eNOS protein did not show any significant difference (Fig. 4). This result suggests that CX3CR1-microglia may be able to inhibit Akt-ERK1/2-nNOS in the NTS of fructose-induced hypertensive rats.

\section{AZD8797 treatment decreases the pro-inflammatory cytokines in the NTS}

To investigate the effect of AZD8797 on chronic proinflammatory environment in the NTS, we analyzed the levels of IL-1 $\beta$, IL-6, TNF- $\alpha$, and fractalkine expression 


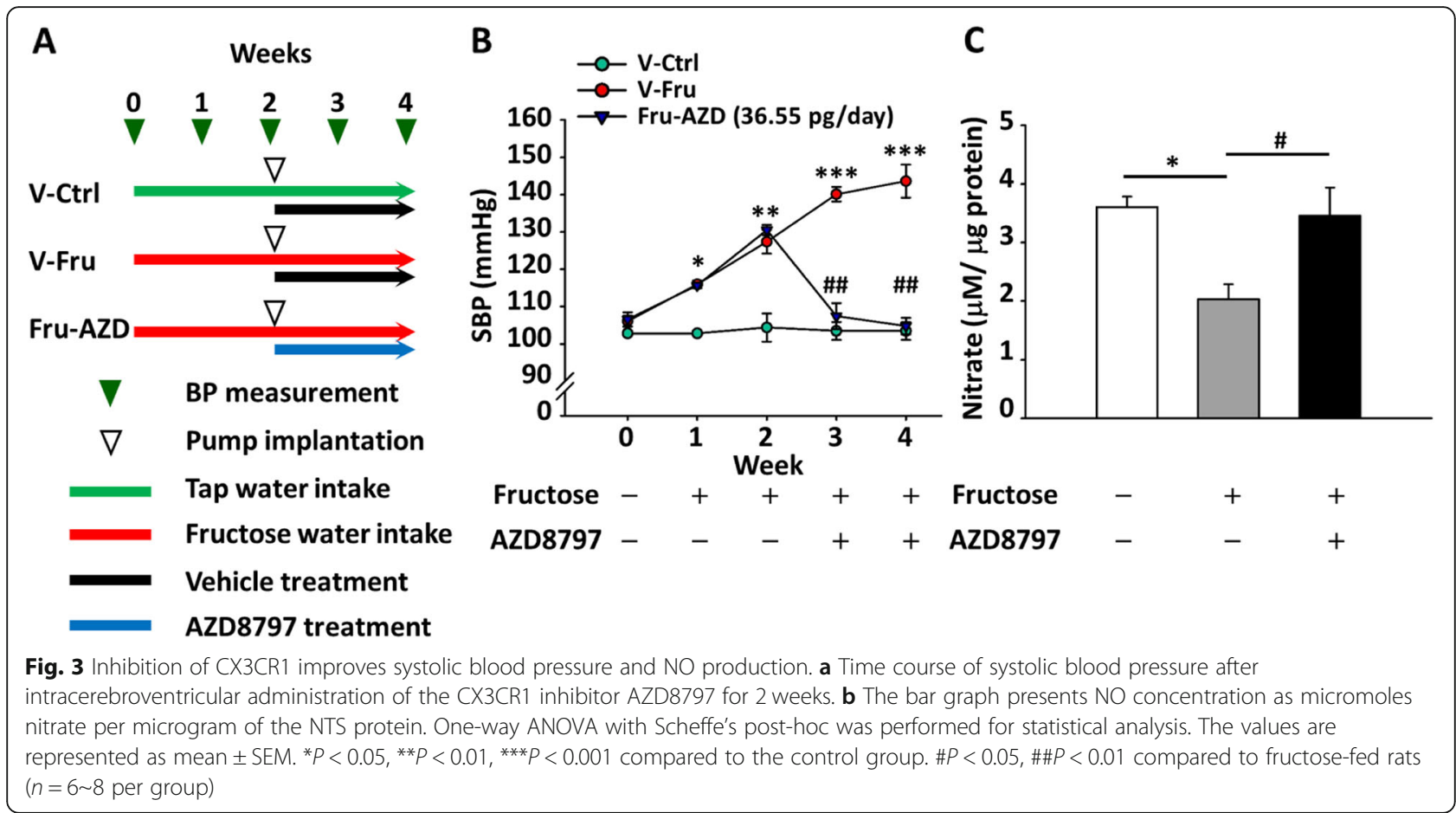

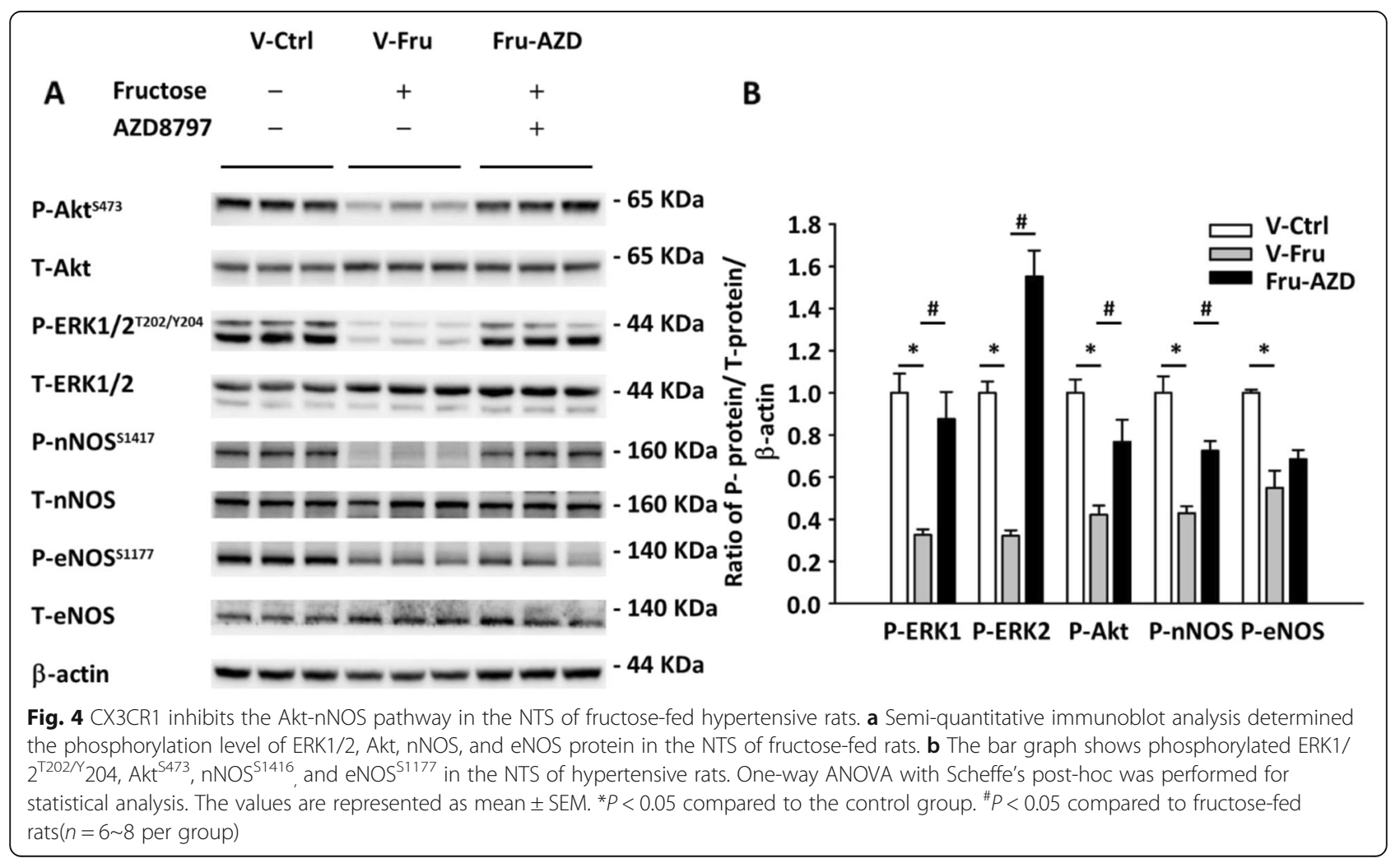


in the NTS by ELISA. The pro-inflammatory cytokines IL-1 $\beta$, IL-6, and TNF- $\alpha$ were decreased in the AZD8797 treatment group. The level of fractalkine was not changed significantly after treatment compared to the control (Table 2). These results indicate that AZD8797 improved the inflammatory environment through CX3CR1microglia inhibition without fractalkine involvement.

Distribution of pro-inflammatory cytokines was assessed by immunohistochemistry (Fig. 5a). As expected, AZD8797 treatment decreases significantly released pro-inflammatory cytokines in the NTS (Fig. 5b), suggesting that the role of CX3CR1-microglia is not only in cardiovascular regulation but also in improving the inflammatory environment of the brainstem.

\section{Discussion}

Although the contribution of the pro-inflammatory factor to hypertension has been increasingly understood, little is known about the functional relation between the pro-inflammatory factor and chronic brain inflammation. In this study, we report that CX3CR1-microglia in the NTS plays a role in fructose-induced hypertension. Inhibited CX3CR1-microglia signaling attenuates fructose-induced hypertension and chronic brain inflammation. The above conclusion is supported by the following observations. ICV administration of AZD8797, a CX3CR1 inhibitor, attenuates fructose-induced hypertension and expression of pro-inflammatory cytokines IL-1 $\beta$, IL- 6 , and TNF- $\alpha$. NO, the gas involved in sympathetic activity and blood pressure regulation in the NTS, was elevated by CX3CR1-microglia inhibition. Furthermore, the pathway important for NO generation, AktnNOS, was restored after CX3CR1-microglia inhibition in the NTS of hypertensive rats. The major finding of this study is that CX3CR1-microglia-mediated downregulation of blood pressure and NO production involve the downregulation of the Akt-nNOS pathway and amelioration of pro-inflammatory cytokines in the NTS of hypertensive rats (Fig. 6).

Pro-inflammatory cytokines produced in the brain play a pathophysiological role in the microenvironment to

Table 2 General characteristics of the fructose-induced WKY rats and AZD8797-treated group

\begin{tabular}{llll}
\hline Parameter/group & V-control & V-fructose & Fru-AZD8797 \\
\hline NTS fractalkine, ng/mg & $5.33 \pm 0.43$ & $8.07 \pm 0.29^{*}$ & $9.52 \pm 0.66^{\#}$ \\
NTS IL-1 $\beta, \mathrm{pg} / \mathrm{mg}$ & $4.83 \pm 0.16$ & $6.93 \pm 0.20^{*}$ & $4.73 \pm 0.13^{\#}$ \\
NTS IL-6, pg/mg & $63.58 \pm 4.32$ & $76.87 \pm 1.34^{*}$ & $49.70 \pm 2.14^{\#}$ \\
NTSTNF-a, pg/mg & $8.16 \pm 0.78$ & $21.63 \pm 2.06^{*}$ & $14.16 \pm 0.65^{\#}$ \\
\hline
\end{tabular}

The values of quantitative ELISA of NTS areas for FKN, IL-1 $\beta, I L-6$, and TNF- $\alpha$ are presented as mean \pm SEM. One-way ANOVA with Scheffe's post-hoc was performed for statistical analysis.

${ }^{*} P<0.05$ vs control group $(n=6),{ }^{\#} P<0.05$ vs fructose group $(n=6 \sim 8$ per group) attract leukocyte or monocyte clusters from the blood vessel to the NTS [26]. Their presence in the NTS suggests that pro-inflammatory cytokines might contribute to cardiovascular diseases, such as hypertension [27]. For example, increase in transcription and protein expression of pro-inflammatory cytokines have been reported in the hippocampus after 2 weeks of $60 \%$ fructose diet intake [4]. Takagishi et al. reported that IL-6 is an endogenous cytokine in the NTS. Their study proposes that IL-6 microinjection has been reported to have attenuated L-glutamate-induced bradycardia in the NTS [28]. This implies that IL-6 overexpression in the NTS may interrupt cardiovascular regulation through baroreceptor afferents' signal transmission. Shi et al. demonstrated that IL-1 $\beta$ stimulates blood pressure increase through ICV injection [29]. Our present observations suggest that fructose feeding promotes early chronic pro-inflammatory cytokines IL- $1 \beta$, IL- 6 , and TNF- $\alpha$ in the NTS.

The FKN and CX3CR1 is a ligand-receptor pair for neuron-microglia communication. Ruchaya et al. reported that FKN and CX3CR1 are abundantly expressed in NTS, and FKN microinjection in the NTS is concerned with cardiovascular regulation [13]. Their findings support the rationale for investigating CX3CR1's role in the NTS of the hypertensive animal model that has brain inflammation. For this reason, we selected AZD8797 to inhibit CX3CR1 for investigating its pharmacological role towards inflammation during hypertension.

Our previous study proposed that NOS function in the NTS may regulate cardiovascular function via PI3KAkt-nNOS and ERK1/2-eNOS pathways [23, 25, 30]. Ruchaya et al. found that FKN regulates cardiovascular function in the NTS through FKN and LY294002 (PI3K-Akt inhibitor) microinjection [13]. AZD8797 is a selective CX3CR1 inhibitor, which has been applied in the therapy of the multiple sclerosis model [11]. We used the ICV method for the delivery of AZD8797. Interestingly, AZD8797 decreases inflammation and increases NO production, which suggests that CX3CR1 in metabolic syndrome is different from its normal state.

FKN is cleaved by metal proteases, such as ADAM10, ADAM17, or MMP-2, which release soluble chemokine domain out of the cells. According to our data, fructose intake induces metal proteases, and the soluble FKN stimulates CX3CR1-expressed cells (Fig. 6). Although this result conflicts with the work previously reported [13], the improvement in inflammation by AZD8797 agrees with the results of Wollberg et al. in that spinal cord inflammation is improved in multiple sclerosis [11].

Previous studies have demonstrated that central inflammation characterizes the hypertensive state and participates in blood pressure elevation [29]. The 


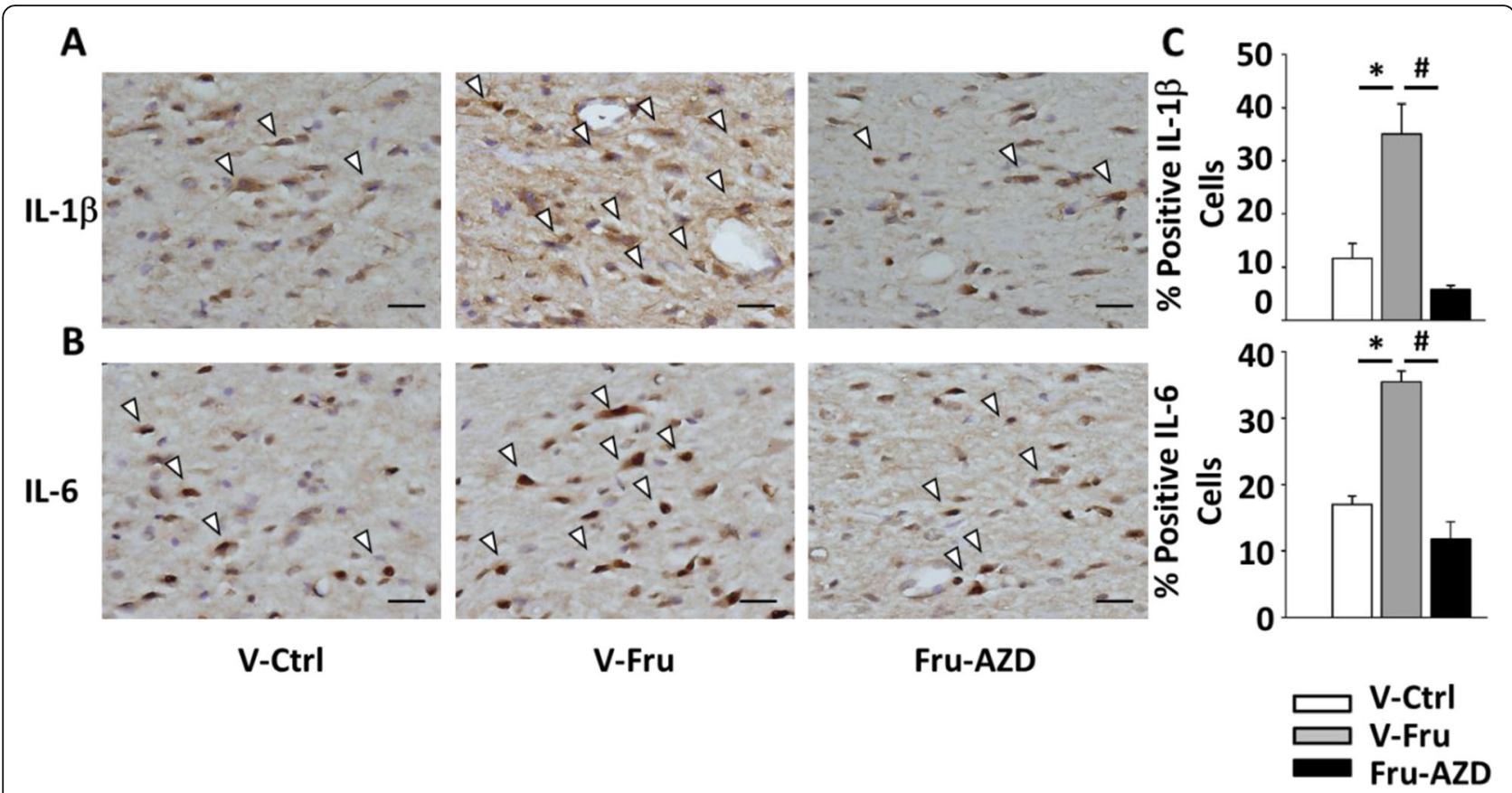

Fig. 5 In situ quantitative immunohistochemical analysis of pro-inflammatory cytokines in the NTS following feeding with 10\% fructose and AZD8797 treatment. a Qualitative analysis of IL-1 $\beta$ and IL-6 were observed by immunohistochemical staining after administration of the CX3CR1 inhibitor AZD8797. The arrowhead indicates positive cells as a representative. The scale bar presents $50 \mu \mathrm{m}$. b Graphs depicting the quantitative analysis of the in situ cytokine-positive cells. The percentage was determined by counting pro-inflammatory positive cells in each hemisphere of the NTS at $200 \times$ magnification. One-way ANOVA with Scheffe's post-hoc was performed for statistical analysis. The values are represented as mean \pm SEM. ${ }^{*} P<0.05$ compared to control rats and ${ }^{\#} P<0.05$ compared to fructose-fed rats ( $n=6 \sim 8$ per group)

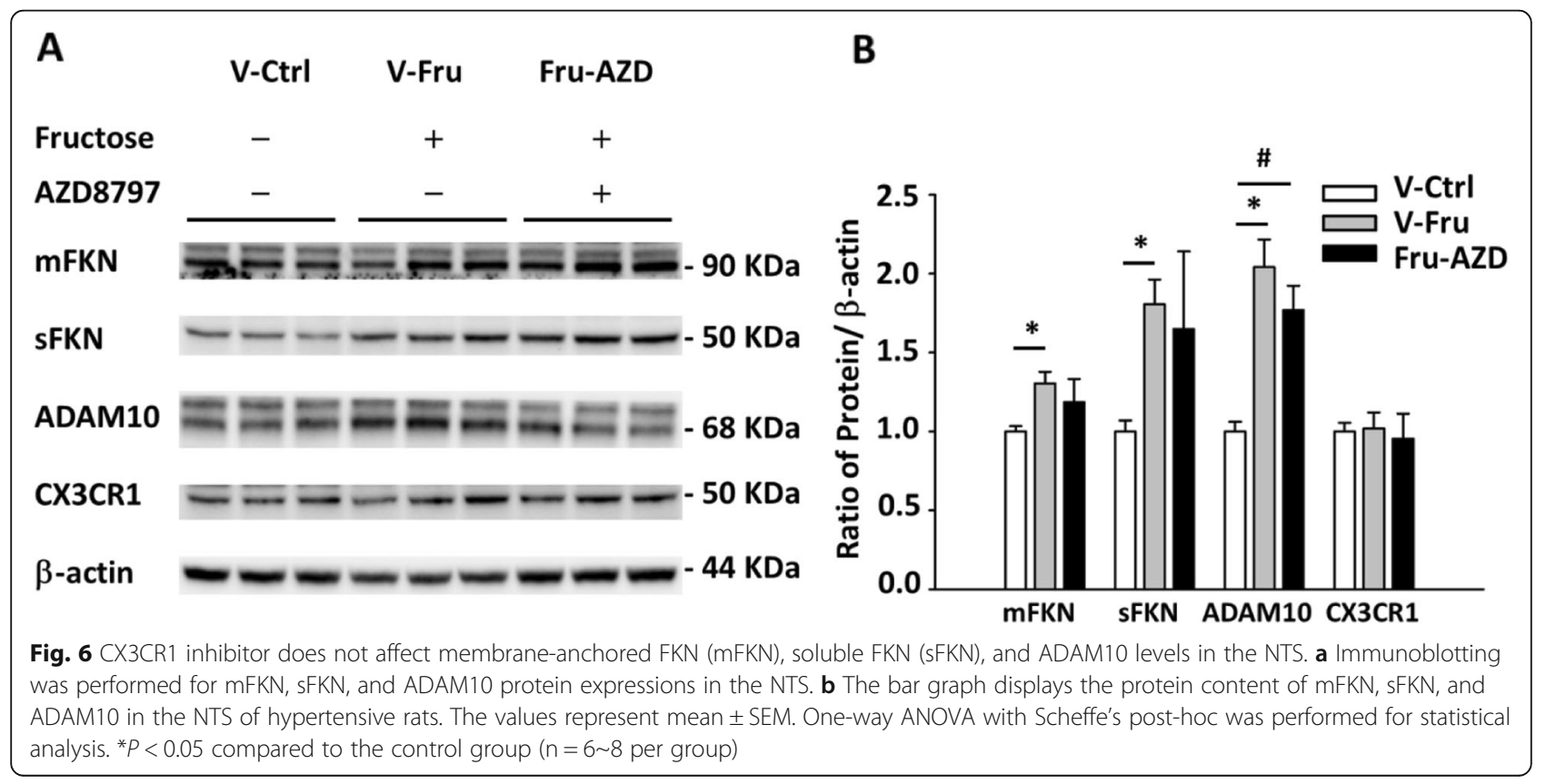


importance of central inflammation in hypertension progression, mortality modulation, and neuronal degeneration disease is now well established by animal models; a theory that Alzheimer's disease is type 3 diabetes has recently been proposed [31, 32]. It has been hypothesized that Alzheimer's disease occurs as a result of decreased NO production in the brain to an elevated, operating pressure [33]. In this study, we investigated the mechanism for CX3CR1-microglia in regards to cardiovascular modulation in the NTS and found that the ERK and Akt cascade, which were initially discovered as essential regulator for cell division and differentiation, in fact, participates in CX3CR1microglia-mediated central cardiovascular regulation. In addition, we also demonstrate that neuronal nitric oxide synthase (nNOS) was mediated by CX3CR1microglia, rather than endothelial nitric oxide synthase (eNOS), which was originally identified in the vascular endothelium and participates in cardiovascular regulation in the NTS. Further investigation of the molecular mechanisms involving blood pressure regulation may unravel the pathogenesis of CX3CR1microglia and brain inflammation in hypertension.

G protein-coupled receptors (GPCRs) play an important role in drug therapy and represent one of the largest families of drug targets for various diseases such as depression and cardiovascular diseases. Previous studies suggested that CX3CR1, a ubiquitously distributed GPCR in the neuron system, participated in blood pressure regulation in the NTS. Insulin resistance-metabolic syndrome patients have been reported to benefit from strategies concerning stimulation of VSMC survival and reduction in FKN/ CX3CR1 signaling to promote plaque stability through an IRS2-dependent signaling [34]. We previously proposed that IRS1 is more critical than IRS2 in insulin resistance-metabolic syndrome of the NTS $[6,35$, 36]. This study suggests that the inhibition of CX3CR1-microglia improves the defective Akt and ERK1/ 2 signaling, both of which are critical pathways for NO production in the NTS. Currently, it potentially appears that improvement in insulin resistance is mediated byCX3CR1-microglia in hypertension progression.

In summary, we showed that AZD8797, a CX3CR1 inhibitor, attenuated fructose-induced hypertension and expression of pro-inflammatory cytokines, IL-1 $\beta$, IL-6, and TNF- $\alpha$. AZD8797 also elevated NO generation, the gas molecule involved in sympathetic activity for regulating blood pressure in the NTS. The CX3CR1-microglia inhibition in turn repaired the Akt-nNOS pathway, an important pathway for $\mathrm{NO}$ generation in the fructoseinduced hypertensive rat (Fig. 7).

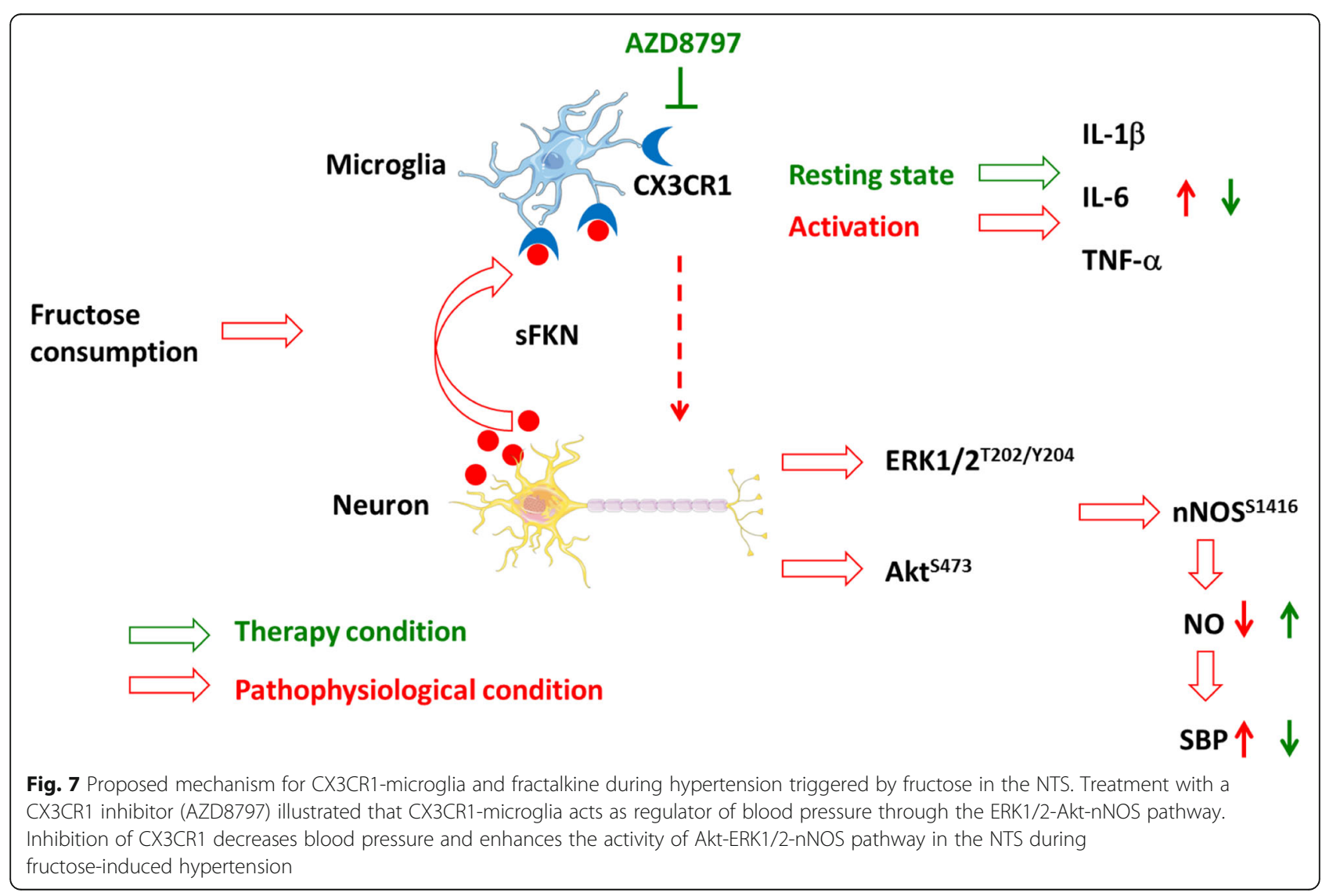




\section{Conclusion}

This study provides the fundamental role of CX3CR1microglia, which can be used for treating cardiovascular disease because these receptors are involved in various pathological conditions. Our novel findings suggest that CX3CR1-microglia may be a potential candidate for treating hypertension and relieving autonomic nerve function through reestablishment of normal crosstalk between the neuron and microglia.

\begin{abstract}
Abbreviations
BP: Blood pressure; CSF: Cerebral spinal fluid; CX3CL1: C-X3-C motif chemokine ligand 1; CX3CR1: C-X3-C motif chemokine receptor 1; dHDL: Direct high-density lipoprotein; FKN: Fractalkine, another term of

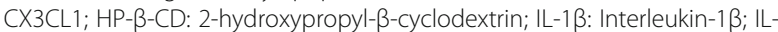
6: Interleukin-6; TNF-a: Tumor necrosis factor-a; ICV: Intracerebroventricular injection; mFKN: Membrane-anchored fractalkine; NO: Nitric oxide; nNOS: Neuronal nitric oxide synthase; NTS: Nucleus tractus solitarii; SBP: Systolic blood pressure; sFKN: Soluble fractalkine; WKY: Wistar-Kyoto rat
\end{abstract}

\section{Acknowledgements}

We thank Dr. Yi-Ling Ma, Ms. Ya-Chu Chuang, Ms. Tzu-Jiun Kuo, and Mr. JuiHsiang Tseng for their excellent technical assistance and laboratory housekeeping during the experimental procedures.

1) What is new?

This study shows that neuroinflammation-regulated neuronal nitric oxide synthase signaling in the nucleus tractus solitarii of hypertensive animals is stimulated by fructose intake. However, the most interesting result of this work is that the CX3CR1 inhibitor reverses the fructose-induced defect in the neuronal nitric oxide synthase signaling pathway by interrupting interaction between fractalkine and CX3CR1.

2) What is relevant?

The crosstalk of neuron-glia involves brainstem autonomic networks during fructose-induced central inflammation. To understand the neuron-glia communication of hypertension, it is critical to develop treatment for neurogenic hypertension. Our study reveals that microglial activity is central to neuroinflammation and neuronal regulation of hypertension, which provides an important aspect to this disease.

Summary

This study provides direct evidence for the CX3CR1 inhibitor in enhancing phosphatidylinositol3-kinase-Akt-neuronal nitric oxide synthase signaling in the nucleus tractus solitarii to prevent fructose-induced hypertension and central inflammation, possibly through the downplay of neuron-glia crosstalk. Our findings provide new insights into the central nervous system in neuron and glia regulations and provide foundation for developing therapies against neurogenic hypertension.

\section{Authors' contributions}

CYH, PWC, YTL, and CJT designed and performed the experiments. PWC, WYH, GCS, YTL, MH, PJL, and CJT funded and supervised the research. CYH and $\mathrm{HHC}$ provided technical and material support for the animal surgery. $\mathrm{CYH}$ analyzed the data. CYH, PWC, and CJT wrote the manuscript. CHY and YTL have full access to all the data in this study and take responsibility for data accuracy and integrity. The authors read and approved the final manuscript.

\section{Funding}

This work was supported by funding from the Ministry of Science and Technology (MOST104-2320-B-075B-003 and MOST-107-2320-B-075B-002) and Kaohsiung Veterans General Hospital (VGHKS106-149, VGHKS106-G01-3, VGHKS107-168, and VGHKS107-G01-1) (to C.-J. T).

\section{Availability of data and materials}

All data generated or analyzed during this study are included in this published article.

\section{Ethics approval and consent to participate}

This study was approved by the Institution Care and Use Committees of Kaohsiung Veterans General Hospital (Kaohsiung, Taiwan).

\section{Consent for publication}

Not applicable

\section{Competing interests}

The authors declared that they have no competing interests.

\section{Author details}

${ }^{1}$ Department of Medical Education and Research, Kaohsiung Veterans General Hospital, Kaohsiung 81300, Taiwan. ${ }^{2}$ Institute of Biomedical Sciences, National Sun Yat-Sen University, Kaohsiung 80424, Taiwan. ${ }^{3}$ Section of Neurology, Kaohsiung Veterans General Hospital, Kaohsiung 81300, Taiwan. ${ }^{4}$ Center for Geriatrics and Gerontology, Kaohsiung Veterans General Hospital, Kaohsiung 81300, Taiwan. ${ }^{5}$ Shu-Zen Junior College of Medicine and Management, Kaohsiung 82144, Taiwan. ${ }^{6}$ Division of General Internal Medicine, Department of Internal Medicine, Kaohsiung Medical University Hospital, Kaohsiung Medical University, Kaohsiung 80708, Taiwan. ${ }^{7}$ Division of Internal Medicine, School of Medicine, College of Medicine, Kaohsiung Medical University, Kaohsiung 80708, Taiwan. ${ }^{8}$ Department of Anesthesiology, Kaohsiung Medical University Hospital, Kaohsiung 80708, Taiwan. ${ }^{9}$ Department of Anesthesiology, Faculty of Medicine, College of Medicine, Kaohsiung Medical University, Kaohsiung 80708, Taiwan.

${ }^{10}$ Genomics Research Center, Academia Sinica, Taipei 11529, Taiwan. ${ }^{11}$ Institute of Clinical Medicine, National Cheng-Kung University, Tainan 70101, Taiwan. ${ }^{12}$ Department of Medical Research, China Medical University Hospital, China Medical University, Taichung 40402, Taiwan.

Received: 27 January 2020 Accepted: 26 May 2020

Published online: 12 June 2020

\section{References}

1. Hajjar I, Kotchen TA. Trends in prevalence, awareness, treatment, and control of hypertension in the United States, 1988-2000. JAMA. 2003;290: 199-206.

2. Williams B. Treating hypertension in patients with diabetes: when to start and how low to go? JAMA. 2015;313:573-74.

3. Sever PS, Dahlöf B, Poulter NR, Wedel H, Beevers G, Caulfield M, et al. Prevention of coronary and stroke events with atorvastatin in hypertensive patients who have average or lower-than-average cholesterol concentrations, in the Anglo-Scandinavian Cardiac Outcomes Trial-Lipid Lowering Arm (ASCOT-LLA): a multicentre randomised controlled trial. Lancet. 2003;361:1149-58.

4. Cigliano L, Spagnuolo MS, Crescenzo R, Cancelliere R, lannotta L, Mazzoli A, et al. Short-term fructose feeding induces inflammation and oxidative stress in the hippocampus of young and adult rats. Mol Neurobiol. 2018;55:286983.

5. Xu M-X, Yu R, Shao L-F, Zhang Y-X, Ge C-X, Liu X-M, et al. Up-regulated fractalkine (FKN) and its receptor CX3CR1 are involved in fructose-induced neuroinflammation: suppression by curcumin. Brain Behav Immun. 2016;58: 69-81.

6. Cheng P-W, Chen Y-Y, Cheng W-H, Lu P-J, Chen H-H, Chen B-R, et al. Wnt signaling regulates blood pressure by downregulating a GSK-3 $\beta$-mediated pathway to enhance insulin signaling in the central nervous system. Diabetes. 2015;64:3413.

7. Limatola C, Ransohoff RM. Modulating neurotoxicity through CX3CL1/ CX3CR1 signaling. Front Cell Neurosci. 2014:8:229.

8. Sheridan GK, Murphy KJ. Neuron-glia crosstalk in health and disease: fractalkine and CX3CR1 take centre stage. Open Biol. 2013;3:130181.

9. Bhaskar K, Konerth M, Kokiko-Cochran ON, Cardona A, Ransohoff RM, Lamb BT. Regulation of tau pathology by the microglial fractalkine receptor. Neuron. 2010;68:19-31

10. Hickman SE, Kingery ND, Ohsumi T, Borowsky M, Wang L-C, Means TK, et al. The microglial sensome revealed by direct RNA sequencing. Nat Neurosci. 2013;16:1896-905.

11. Ridderstad Wollberg A, Ericsson-Dahlstrand A, Jureus A, Ekerot P, Simon S, Nilsson $M$, et al. Pharmacological inhibition of the chemokine receptor CX3CR1 attenuates disease in a chronic-relapsing rat model for multiple sclerosis. Proc Natl Acad Sci U S A. 2014;111:5409-14.

12. Poniatowski LA, Wojdasiewicz P, Krawczyk M, Szukiewicz D, Gasik R, Kubaszewski $\mathrm{L}$, et al. Analysis of the role of $\mathrm{CX} 3 \mathrm{CL} 1$ (fractalkine) and its receptor CX3CR1 in traumatic brain and spinal cord injury: insight into 
recent advances in actions of neurochemokine agents. Mol Neurobiol. 2017; 54:2167-88.

13. Ruchaya PJ, Paton JF, Murphy D, Yao ST. A cardiovascular role for fractalkine and its cognate receptor, CX3CR1, in the rat nucleus of the solitary tract. Neuroscience. 2012;209:119-27.

14. Tseng CJ, Liu HY, Lin HC, Ger LP, Tung CS, Yen MH. Cardiovascular effects of nitric oxide in the brain stem nuclei of rats. Hypertension. 1996;27:36-42.

15. Krum H, Schlaich MP, Sobotka PA, Böhm M, Mahfoud F, Rocha-Singh K, et al. Percutaneous renal denervation in patients with treatment-resistant hypertension: final 3-year report of the Symplicity HTN-1 study. Lancet. 2014;383:622-9.

16. Bray GA, Popkin BM. Dietary sugar and body weight: Have we reached a crisis in the epidemic of obesity and diabetes? Diabetes Care. 2014;37:950

17. Tappy L, Lê K-A. Metabolic effects of fructose and the worldwide increase in obesity. Physiol Rev. 2010;90:23.

18. Lopes A, Vilela TC, Taschetto L, Vuolo F, Petronilho F, Dal-Pizzol F, et al. Evaluation of the effects of fructose on oxidative stress and inflammatory parameters in rat brain. Mol Neurobiol. 2014;50:1124-30.

19. Glushakova O, Kosugi T, Roncal C, Mu W, Heinig M, Cirillo P, et al. Fructose induces the inflammatory molecule ICAM-1 in endothelial cells. J Am Soc Nephrol. 2008;19:1712-20.

20. Oudot C, Lajoix $A D$, Jover $B$, Rugale $C$. Dietary sodium restriction prevents kidney damage in high fructose-fed rats. Kidney Int. 2013;83: 674-83.

21. Yeh TC, Liu CP, Cheng WH, Chen BR, Lu PJ, Cheng PW, et al. Caffeine intake improves fructose-induced hypertension and insulin resistance by enhancing central insulin signaling. Hypertension. 2014;63:535-41.

22. Cheng PW, Ho WY, Su YT, Lu PJ, Chen BZ, Cheng WH, et al. Resveratrol decreases fructose-induced oxidative stress, mediated by NADPH oxidase via an AMPK-dependent mechanism. Br J Pharmacol. 2014;171:2739-50.

23. Huang HN, Lu PJ, Lo WC, Lin CH, Hsiao M, Tseng CJ. In situ Akt phosphorylation in the nucleus tractus solitarii is involved in central control of blood pressure and heart rate. Circulation. 2004;110:2476-83.

24. Paxinos $G$, Watson $C$. The rat brain in stereotaxic coordinates. 6th ed. San Diego: Academic Press; 2007

25. Cheng WH, Lu PJ, Ho WY, Tung CS, Cheng PW, Hsiao M, et al. Angiotensin II inhibits neuronal nitric oxide synthase activation through the ERK1/2-RSK signaling pathway to modulate central control of blood pressure. Circ Res. 2010;106:788-95.

26. Waki H, Hendy EB, Hindmarch CC, Gouraud S, Toward M, Kasparov S, et al. Excessive leukotriene B4 in nucleus tractus solitarii is prohypertensive in spontaneously hypertensive rats. Hypertension. 2013;61:194-201.

27. Shi P, Raizada MK, Sumners C. Brain cytokines as neuromodulators in cardiovascular control. Clin Exp Pharmacol Physiol. 2010;37:e52-7.

28. Takagishi M, Waki H, Bhuiyan MER, Gouraud SS, Kohsaka A, Cui H, et al. II-6 microinjected in the nucleus tractus solitarii attenuates cardiac baroreceptor reflex function in rats. Am J Physiol Regul Integr Comp Physiol. 2009;298: R183-R90.

29. Shi P, Diez-Freire C, Jun JY, Qi Y, Katovich MJ, Li Q, et al. Brain microglial cytokines in neurogenic hypertension. Hypertension. 2010;56:297-303.

30. Ho WY, Lu PJ, Hsiao M, Hwang HR, Tseng YC, Yen MH, et al. Adenosine modulates cardiovascular functions through activation of extracellular signal-regulated kinases 1 and 2 and endothelial nitric oxide synthase in the nucleus tractus solitarii of rats. Circulation. 2008;117:773-80,

31. de la Monte SM, Wands JR. Alzheimer's disease is type 3 diabetes-evidence reviewed. J Diabetes Sci Technol. 2008;2:1101-13.

32. Mittal K, Mani RJ, Katare DP. Type 3 diabetes: cross talk between differentially regulated proteins of type 2 diabetes mellitus and Alzheimer's disease. Sci Rep. 2016;6:25589.

33. Kruyer A, Soplop N, Strickland S, Norris EH. Chronic hypertension leads to neurodegeneration in the TgSwDI mouse model of Alzheimer's disease. Hypertension. 2015;66:175-82.

34. Martinez-Hervas S, Vinue A, Nunez L, Andres-Blasco I, Piqueras L, Real JT, et al. Insulin resistance aggravates atherosclerosis by reducing vascular smooth muscle cell survival and increasing CX3CL1/CX3CR1 axis. Cardiovasc Res. 2014:103:324-36.

35. Cheng PW, Kang BH, Lu PJ, Lin SS, Ho WY, Chen HH, et al. Involvement of two distinct signalling pathways in IGF-1-mediated central control of hypotensive effects in normotensive and hypertensive rats. Acta Physiol (Oxf). 2014;212:28-38.
36. Cheng PW, Lin YT, Ho WY, Lu PJ, Chen HH, Lai CC, et al. Fructose induced neurogenic hypertension mediated by overactivation of p38 MAPK to impair insulin signaling transduction caused central insulin resistance. Free Radic Biol Med. 2017;112:298-307.

\section{Publisher's Note}

Springer Nature remains neutral with regard to jurisdictional claims in published maps and institutional affiliations.
Ready to submit your research? Choose BMC and benefit from:

- fast, convenient online submission

- thorough peer review by experienced researchers in your field

- rapid publication on acceptance

- support for research data, including large and complex data types

- gold Open Access which fosters wider collaboration and increased citations

- maximum visibility for your research: over $100 \mathrm{M}$ website views per year

At BMC, research is always in progress.

Learn more biomedcentral.com/submissions 